\title{
QUANTIFYING THE UTILITY OF THE PAST IN MINING LARGE DATABASES+
}

\author{
VIKRAM PUDI ${ }^{1}$ and JAYANT R. HARITSA ${ }^{12}$ \\ ${ }^{1}$ Database Systems Lab, SERC, Indian Institute of Science, Rangalore 560012. INI)[A \\ ${ }^{2}$ Database Systems Research Department, Lucent Bell Labs, 600 Mountain Avenue, Murray Hill, N.J 07974. USA
}

\begin{abstract} utilizing previou Incremental mining algorithms that can efficiently derive the current mining output by mining results are attractive to business organizations since data mining is typically bust and efficient incremental mining of association rules on large market basket databases. DELTA guarantees efficiency by ensuring that, for any dataset, at most three passes over the increment and one pass over the previous database are required to generate the desired rules. Further, it. handles "multi-support" environments where the support requirements for the current mining differ from those used in the previous mining, a feature in tune with the exploratory nature of the mining process. We present a performance evaluation of DELT.4 on large databases over a range of increment sizes and data distributions, as well as change in support requirements. The experimental results show that DELTA can provide significant improvements in execution times over previously proposed incremental algorithms in all these environments. In fact, for many workloatis, its performance is close to that, achieved by an optimal, but practically infeasible, algorithm.
\end{abstract}

Key words: Data Mining, Association Rule. Hierarchical Association Rule, Incremental Mining

\section{INTRODUCTION}

In many business organizations, the historical database is dynamic in that, it is periodically updated with fresh data. For such environments, data mining is not a one-time operation but a recurring activity, especially if the database has been significantly updated since the previous mining exercise. Repeated mining may also be required in order to evaluate the effects of business strategies that have been implemented based on the results of the previous mining. In an overall sense, mining is essentially an exploratory activity and therefore, by its very nature, operates as a feedback process wherein each new mining is guided by the results of the previous mining.

In the above context, it is attractive to consider the possibility of using the results of the previous mining operations to minimize the amount of work done during each new mining operation. That, is. given a previously mined database $D B$ and a subsequent increment $d b$ to this database, to efficiently mine $d b$ and $D B \cup d b$. Mining $d b$ is necessary to evaluate the effects of business strategies; whereas mining $D B \cup d b$ is necessary to maintain the updated set of mining rules. Such "incremental" mining is the focus of this paper. Practical applications where incremental mining techniques are especially useful include data warehouses and Web mining since these systems are constantly updated with fresh data - on the web, for instance, about, one million pages are added daily [12].

We consider here the design of incremental mining algorithms for databases that can be rep-resented as a two-dimensional matrix, with one dimension being a (fixed) set of possibilities, and the other dimension being a dynamic history of instances, with each instance recording a joint occurrence of a subset of these possibilities. A canonical example of such a matrix is the "market basket" database [1], wherein the possibilities are the items that are on sale by the store, and the instances record the specific set of items bought by each customer. For ease of exposition. we will assume a market basket database in the remainder of this paper.

Within the above framework, we focus in particular on the identification of association rules [1], that is, rules which establish interesting correlations about the joint, occurrence of items in customer

${ }^{\dagger}$ Recommended by Felipe Carino 
purchases - for example, "Eighty percent of the customers who bought milk also bought cereal". Association rules have been productively used in a variety of applications ranging from bookstores (e.g. when you purchase a book at Amazon.com, the site informs you of the other books that have been typically bought by earlier purchasers of your book) to health care (e.g. in detecting shifts in infection and antimicrobial resistance patterns in intensive care units [7]).

Since the association rule problem is well-established in the database research literature (see $[1,19]$ for complete details), we assume hereon that the reader is familiar with the concepts and algorithmic techniques underlying these rules. In particular, we assume complete knowledge of the classical Apriori algorithm [3].

\subsection{The State-of-the-Art}

The design of incremental mining algorithms for association rules has been considered earlier in $[5,8,9,10,11,18]$. While these studies were a welcome first step in addressing the problem of incremental mining, they also suffer from a variety of limitations that make their design and evaluation unsatisfactory from an "industrial-strength" perspective:

Effect of Skew The effect of temporal changes (i.e. skew) in the distribution of database values between DB and $d b$ has not been considered. However, in practical databases, we should typically expect to see skew for the following reasons: (a) inherent seasonal fluctuations in the business process, and/or (b) effects of business strategies that have been put into place since the last mining. So, we expect that skew would be the norm, rather than the exception.

As we will show later in this paper, the performance of the algorithms presented in $[11,18]$ is sensitive to the skew factor. In fact, their sensitivity is to the extent that, with significant skew and substantial increments, they may do worse than even the naive approach of completely ignoring the previous mining results and applying Apriori from scratch on the entire current database.

Size of Database The evaluations of the algorithms has been largely conducted on databases and increments that are small relative to the available main memory. For example, the standard experiment considered a database with $0.1 \mathrm{M}$ tuples, with each tuple occupying approximately 50 bytes, resulting in a total database size of only $5 \mathrm{MB}$. For current machine configurations, this database would completely fit into memory with plenty still left to spare. Therefore, the ability of the algorithms to scale to the enormous disk-resident databases that are maintained by most business organizations, has not been clearly established.

Characterizing Efficiency Apart from comparing their performance with that of Apriori, no quantitative assessment has been made of the efficiency of these algorithms in terms of their distance from the optimal, which would be indicative of the scope, if any, for further improvement in the design of incremental algorithms.

Incomplete Results Almost all the algorithms fail to provide the mining results for solely the increment, $d b$. As mentioned before, these results are necessary to help evaluate the effects of business strategies that have been put into place since the previous mining.

Changing User Requirements It is implicitly assumed that the minimum support specified by the user for the current database $(D B \cup d b)$ is the same as that used for the previously mined database $(D B)$. However, in practice, given mining's exploratory nature, we could expect user requirements to change with time, perhaps resulting in different minimum support levels across mining operations. Extending the algorithms to efficiently handle such "multisupport" environments is not straightforward.

Hierarchical Databases Virtually all the previous work is applicable only to "flat" databases where the items in the database have independent characteristics. In reality, however, databases are often "hierarchical", that is, there exists a is-a hierarchy over the set of items 
in the database ${ }^{\dagger}$. For example, sweaters and ski, jackets are both instances of winter wear. As pointed out in [16], such taxonomies are valuable since rules at lower levels may riot, satisfy the minimum support threshold and hence not, many rules arc likely to be output if they are not considered. They are also useful to prune away many of the uninteresting rules that would be output at low minimum support thresholds: since a significant fraction of the rules at lower levels may be subsumed by those at higher levels.

\section{1.t. Contributions}

In this paper, we present. and evaluate an incremental mining algorithm called DELTA (Differential Evaluation of Large iTemset Algorithm). The core of DELTA is similar to the previous algorithms but it also incorporates important design alterations for addressing their above-mentioned limitations. With these extensions, DELTA represents a practical algorithm that can be effectively utilized for real-world databases. The main features of the design and evaluation of DELTA are the following:

- DELTA guarantees that,,for the entire mining process, at most three passes over tlir increment and one pass over the previous database may be necessary. We expect that such bounds will be useful to businesses for the proper scheduling of their mining operations.

- For the special case wliere the new results are a subset of the old results, anti therefore in principle roquiring no processing over the previous database. DELTA is optimal in that it, requires only a single pass over the increment to complete the mining process.

- For computing the negative border [19] closure, a major performance-detcrmining factor in the incremental mining process, a new hybrid scheme that combines the features of earlior approaches is implemented.

- DELTA provides complete mining results for both the entire current database as well as solely tlir increment.

- DELTA can handle multi-support environments. requiring only one additional pass over the current database to achieve this functionality.

- By carefully integrating optimizations previously proposed for first-time hierarchical mining algorithms, the DELTA design has been extended to efficiently handle incremental mining of hierarchical association rules.

- The performance of DELTA is evaluated on a variety of dynamic databases and compared with that of Apriori and the previously proposed incremental mining algorithms for flat, association rules. For hierarchical association rules. we compare DELTA against, the Cumulate first-time mining algorithm presented in [16]. All experiments are made on databases that arc significantly larger than the entire main memory of the machine on which the experiments were conducted. The effects of database skew are also modeled.

The results of our experiments show that DELTA can provide significant improvements in execution times over the previous algorithms in all these environments. Further. DELTA's performance is comparatively robust, with respect, to database skew.

- We also include in our evaluation suite the performance of an an oracle that has complets apriori knowledge of the identities of all the large itemsets (and their associated negative border) both in the current database as well as in the increment and only requires to find their respective counts. It is easy to see that this algorithm, although practically infcasiblr. represents the optimal since all other algorithms will have to do at least the same amount of work. Therefore, modeling the oracle's performance permits us to characterize the efficiency of practical algorithms in terms of their distance from the optimal.

\footnotetext{
†Fiat and hierarchical databases are also referred to as "boolean" and "generalized" databases, respectively, in the mining literature.
} 


\begin{tabular}{|l|l|}
\hline$D B, d b, D B \cup d b$ & Previous, increment, and current database \\
$s u p_{\tilde{h}} \bar{c}, s u p D B \cup d b$ & Previous and New Minimum Support Thresholds \\
$s u p_{m i n}$ & Minimum Support Threshold when $s u p_{\min }^{D B}=s u p_{\min }^{D B \cup d b}$ \\
$L^{B B}, E^{2 b}, L^{D B \cup d b}$ & Set of large itemsets in $D B, d b$ and $D B \cup d b$ \\
$N^{D B}, N^{d b}, N^{D B \cup d b}$ & Negative borders of $L^{D B}, L^{d b}$ and $L^{D B \cup d b}$ \\
$L$ & $L^{D B \cup d b} \cap\left(L^{D B} \cup N^{D B}\right)$ \\
$N$ & Negative border of $L$ \\
Small & Set of small itemsets with known counts (during program execution) \\
\hline
\end{tabular}

Table 1: Notation

Our experiments show that DELTA's efficiency is close to that obtained by the oracle for many of the workloads considered in our study. This shows that DELTA is able to extract most of the potential for using the previous results in the incremental mining process.

\subsection{Organization}

The remainder of this paper is organized as follows: The DELTA algorithm for both flat and hierarchical association rules is presented in Section 2 for the equi-support environment. The algorithm is extended to handle the multi-support case in Section 3. The previously proposed incremental mining algorithms are summarized in Section 4. The performance model is described in Section 5 and the results of the experiments are highlighted in Section 6. Finally, in Section 7, we present the conclusions of our study and outline future research avenues.

\section{THE DELTA ALGORITHM}

In this section, we present the design of the DELTA algorithm. For ease of exposition, we first consider the "equi-support" case, and then in Section 3, we describe the extensions required to handle the "multi-support" environment. In the following discussion and in the remainder of this paper, we use the notation given in Table 1. Also, we use the terms "large", "small", "count" and "support" with respect to the entire database $\boldsymbol{D} \boldsymbol{B} \cup d b$, unless otherwise mentioned.

The input to the incremental mining process consists of the set of previous large itemsets $L^{D B}$, its negative border $N^{D B}$, and their associated supports. The output is the updated versions of the inputs, namely, $L^{D B \cup d b}$ and $N^{D B \cup d b}$ along with their supports. In addition, the mining results for solely the increment, namely, $L^{d b} \cup N^{d b}$, are also output.

\subsection{The Mechanics of DELTA}

The pseudo-code of the core DELTA algorithm for generating flat association rules is shown in Figure 1-the extension to hierarchical association rules is presented in Section 2.2. At most three passes over the increment and one pass over the previous database are made, and we explain below the steps taken in each of these passes. After this explanation of the mechanics of the algorithm, we discuss in Section $\mathbf{2 . 3}$ the rationale behind the design choices.

\subsubsection{First Pass over the Increment}

In the first pass, the counts of itemsets in $L^{D B}$ and $N^{D B}$ are updated over the increment $d b$, using the function UpdateCounts (line 1 in Figure 1). By this, some itemsets in $N^{D B}$ may become large and some itemsets in $L^{D B}$ may become small. Let the resultant set of large itemsets be $L$. These large itemsets are extracted using the function GetLarge (line 2). The remaining itemsets are put in Small (line 3), and are later used for pruning candidates. The algorithm terminates if no itemsets have moved from $N^{D B}$ to $L$ (lines 4-5). This is valid due to the following Theorem presented in [18]: 


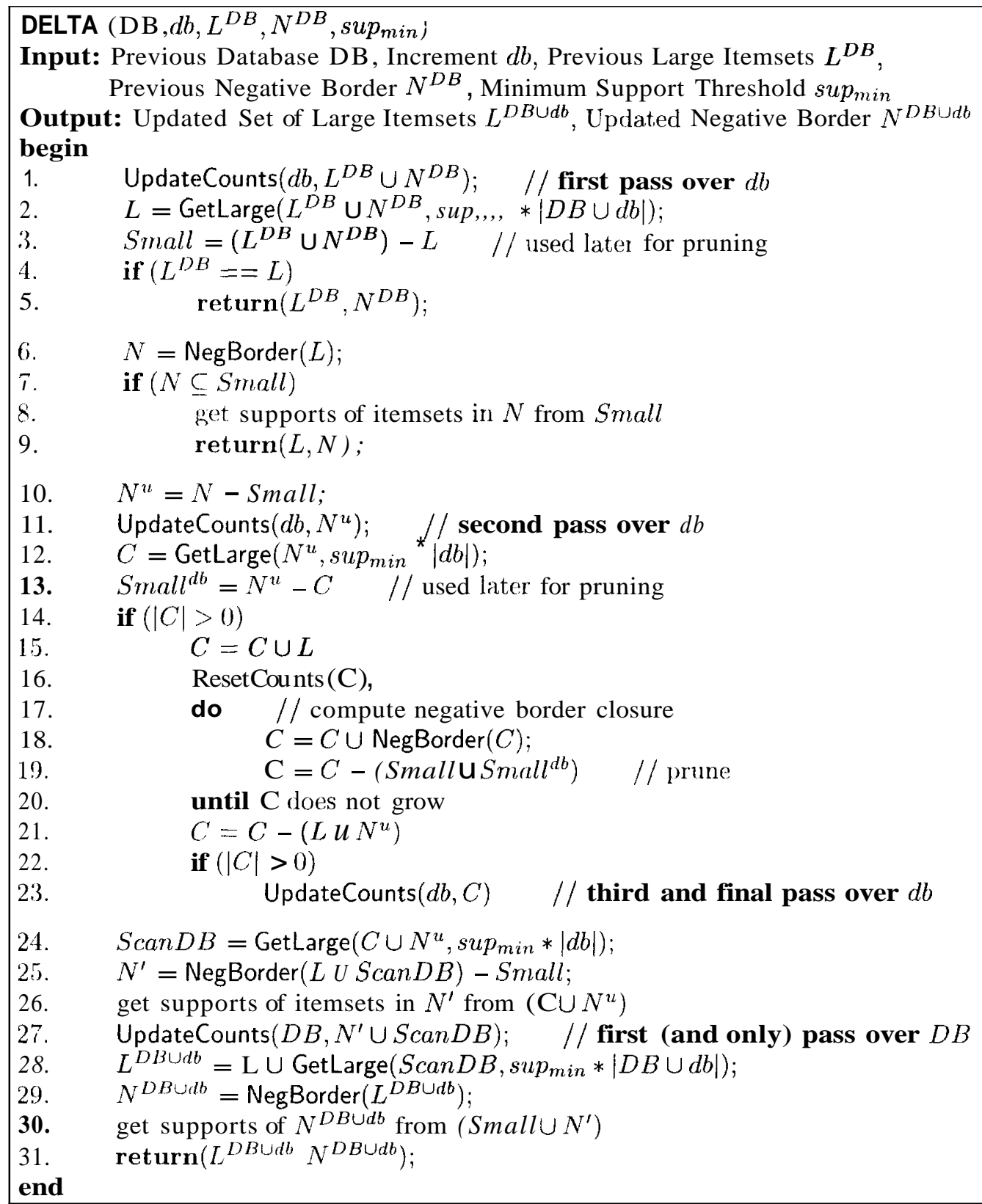

Fig. 1: The DELTA Incremental Mining Algorithm

Theorem 1 If $X$ is an itemset that is not in $L^{D B}$ but is in $L^{D B \cup d b}$, then there must be some subset $x$ of $X$ which was in $N^{D B}$ and is now in $L^{D B \cup d b}$.

Hence, for the special case where the new results are a subset of the old results, and therefore in principle requiring no processing over the previous database, DELTA is optimal in that it requires only a single pass over the increment to complete the mining process.

\subsubsection{Second Pass over the Increment}

On the other hand, if some itemsets do move from $N^{D B}$ to $\mathrm{L}$, then the negative border $N$ of $I$, is computed (line $G$ ), using the AprioriGen [3] function. Itemsets in $N$ with unknown counts are stored in a set $N^{u}$ (line 10). The remaining itemsets in $N$ i.e. with known counts, are all small. 
Therefore, the only itemsets that may be large (and are not yet known to be so) are those in $N^{u}$ and their extensions. If there are no itemsets in $N^{u}$, the algorithm terminates (lines 7-9).

Now, any itemset in $N^{u}$ that is not locally large in $d b$ cannot be large in $D B \cup d b$. Further, none of its extensions can be large as well. This is based on the following observation of $[8]^{\dagger}$ :

Theorem 2 An itemset can be present in $L^{D B \cup d b}$ only if it is present in either $L^{D B}$ or $L^{d b}$ (or both).

Therefore, a second pass over the increment is made to find the counts within $d b$ of $N^{u}$ (line 11). Those itemsets that turn out to be small in $d b$ are stored in a set called Small $^{d b}$ (line 13 ), which is later used for pruning candidates.

\subsubsection{Third (and Final) Pass over the Increment}

We then form all possible extensions of $L$ which could be in $L^{D B \cup d b} \cup N^{D B \cup d b}$ and store them in set $\mathrm{C}$. This is done by computing the remaining layers of the negative border closure of $L$ (lines 15-20). (We expect that the remaining layers can be generated together since the number of 2-itemsets in $L$ is typically much smaller than the overall number of all possible 2-itemset pairs.) At the start of this computation, the counts of itemsets in $C$ are reset to zero using the function ResetCounts (line 16). Then, at every stage during the computation of the closure, those itemsets that are in Small and Small ${ }^{d b}$ are removed so that none of their extensions are generated (line 19). After all the layers are generated, itemsets from $L$ and $N^{u}$ are removed from $C$ since their counts within $D B \cup d b$ and $d b$ respectively, are already available (line 21). The third and final pass over $d b$ is then made to find the counts within $d b$ of the remaining itemsets in $C$ (line 23).

\subsubsection{First (and Only) Pass over the Previous Database}

Those itemsets of the closure which turn out to be locally large in $d b$ need to be counted over $D B$ as well to establish whether they are large overall. We refer to these itemsets as $S c a n D B$ (line 24). Since the counts of $N^{D B \cup d b}$ need to be computed as well, we evaluate $\operatorname{NegBorder}(L \cup S c a n D B)$. From this the itemsets in Small are removed since their counts are already known. The counts of the remaining itemsets (i.e. $N^{\prime}$ in line 25) are then found by making a pass over $D B$ (line 27).

After the pass over $D B$, the large itemsets from $S \operatorname{can} D B$ are gathered to form $L^{D B \cup d b}$ (line 28) and then its negative border $N^{D B \cup d b}$ is computed (line 29). The counts of $N^{D B \cup d b}$ are obtained from Small and $N^{\prime}$ (line 30). Thus we obtain the final set of large itemsets $L^{D B \cup d b}$ and its negative border $N^{D B \cup d b}$.

\subsubsection{Results for the Increment}

Performing the above steps results in the generation of $L^{D B \cup d b}$ and $N^{D B \cup d b}$ along with their supports. But, as mentioned earlier, we also need to generate the mining results for solely the increment, namely, $L^{d b} \cup N^{d b}$. To achieve this, the following additional processing is carried out during the above-mentioned passes:

After the first pass over the increment, we have the updated counts of all the itemsets in $L^{D B} \cup N^{D B}$. Therefore, the counts of these itemsets with respect to the increment alone is very easily determined by merely computing the differences between the updated counts and the original counts. After this computation, the itemsets that turn out to be large within $d b$ are gathered together and their negative border is computed.

If the counts within $d b$ of some itemsets in the negative border are unknown, these counts are determined during the second pass over the increment. Subsequently, the negative border closure of the resultant large itemsets (over $d b$ ) is computed and the counts within $d b$ of the itemsets in the closure are determined during the third pass over the increment. Finally, the identities and counts within $d b$ of itemsets in $L^{d b} \cup N^{d b}$ are extracted from the closure.

\footnotetext{
†This observation applies only to the equi-support case.
} 
In the above, note that a particular itemset could be a candidate for computing $L^{d l b} \mathrm{u} N^{d b}$, as well as $L^{D B \cup d b} \cup N^{D B \cup d h}$. To ensure that there is no unnecessary duplicate counting, all such common itemsets are identificd and two counters are maintained for each of them: the first,counter initially stores the itemset's support in $D B$, while the second stores the support, in $d b$. After the support in $d b$ is computed, the first counter is incremented by this value it then reflects the support in $D B \mathrm{U} d b$.

\subsection{Generating Hierarchical Association Rules}

The processirig steps described in the previous sub-section are conipletely sufficient to deliver the desired mining outputs for flat databases. We now niove on to describing how it is easily possiblti to extend the DELTA design to also handle the generation of association rules for hierarchical databases.

The hierarchical rule mining problem is to find association rules between items at any level of a given taxonomy graph (is-a hierarchy). An obvious but, inefficient solution to this problem is to reduce it, to a.flat mining context using the following strategy: While reading each transaction from the database. dynamically create an "augmented" transaction that also includes all thr ancestors of all the items featured in the original transaction. Now. any of the flat, mining algorithms can bo applied on this augmented database.

A set of optimizations to improve upon the above scheme were introduced in [16] as part, of the Cumulate (first,-time) hierarchical mining algorithm. Interestingly, we have found that, these optimizations can be utilized for incremental mining as well, and in particular, can be cleanly integrated in the core DELTA algorithm. In the remainder of this sub-section, we describe the optimizations and their incorporation in DELTA.

\subsubsection{Cumulate Optimizations}

Cumulate's optimizations for efficiently mining hierarchical databases are the following:

o Pre-computing ancestors Rather than finding the ancestors for each item by traversing the taxonomy graph, the ancestors for each item arc precomputed and stored in an array.

$o$ Filtering the ancestors added to transactions While reading a transaction from the database, it is riot necessary to augment it with all ancestors of items in that transaction. Only ancestors of items in the transaction that are also present in some candidate itemset, are added.

o Pruning itemsets containing an item and its ancestor A candidate itemset, that contains both an item and its ancestor may be pruned. This is because it will have exactly the same support as the itemset which doesn't contain that ancestor and is therefore redundant.

\subsubsection{Incorporation in DELTA}

The above optimizations are incorporated in DELTA in the following manner:

1. The first optimization is performed only in routines that access the database and therefore do not affect the structure of the DELTA algorithm.

2. The second optimization is performed before each pass over the increment or previous database. Ancestors of items that are not, part of any candidate are removed from the arrays of ancestors that were precomputed during the first optimization.

3. The third optimization is performed only once and that is at, the end of the first pass over the increment. At this stage the identities of all potentially large 2-itemsets (over $D B \cup d b$ ) are known, and hence no further candidate 2-itemsets will be generated. Among the potentially large 2-itemsets, those that contain an item and its ancestor are pruned. It follows that candidates generated from the remaining 2 -iternsets will also have the same property, i.e. 
they will not contain an item and its ancestor. Hence this optimization does not need to be applied again.

\subsection{Rationale for the DELTA Design}

Having described the mechanics of the DELTA design, we now provide the rationale for its construction:

Let $L$ be the set of large itemsets in $L^{D B} \cup N^{D B}$ that survive the support requirement after their counts have been updated over $d b$, and $N$ be its negative border. Now, if the counts of all the itemsets in $N$ are available, then the final output is simply $L \cup N$. Otherwise, the only itemsets that may be large (and are not yet known to be so) are those in $N$ with unknown counts and their extensions - by virtue of Theorem 1. At this juncture, we can choose to do one of the following:

Complete Closure Generate the complete closure of the negative border, that is, all extensions of the itemsets in $N$ with unknown counts. While generating the extensions, itemsets that are known to be small may be removed so that none of their extensions are generated. After the generation process is over, find the counts of all the generated itemsets by performing one scan over $D B \cup d b$. We now have all the information necessary to first identify $L^{D B \cup d b}$, and then the associated $N^{D B \cup d b}$.

Layered Closure Instead of generating the entire closure at one shot, generate the negative border "a layer at a time". After each layer is computed, update the counts of the itemsets in the layer by performing a scan over $D B \cup d b$. Use these counts to prune the set of itemsets that will be used in the generation of the next layer.

Hybrid Closure A combination of the above two schemes, wherein the closure is initially generated a layer at a time, and after a certain number of layers are completed, the remaining complete closure is computed. The number of layers upto which the closure is generated in a layered manner is a design parameter.

The first scheme, Complete Closure, appears infeasible because it could generate a very large number of candidates if the so-called "promoted borders" [11], that is, itemsets that were in $N^{D B}$ but have now moved to $L^{D B \cup d b}$, contain more than a few 1 -itemsets. This is because if $p_{1}$ is the number of 1-itemsets in the promoted borders, a lower bound on the number of candidates is $2^{p_{1}}\left(|L|-p_{1}\right)$. This arises out of the fact that every combination of the $p_{1} 1$-itemsets is a possible extension, and all of them can combine with any other large itemset in $L$ to form candidates. Therefore, even for moderate values of $p_{1}$, the number of candidates generated could be extremely large.

The second strategy, Layered Closure, avoids the above candidate explosion problem since it brings a pruning step into play after the computation of each layer. However, it has its own performance problem in that it may require several passes over the database, one per layer, and this could turn out to be very costly for large databases. Further, it becomes impossible to provide bounds on the number of passes that would be required for the mining process.

Therefore, in DELTA, we adopt the third hybrid strategy, wherein an initial Layered Closure approach is followed by a Complete Closure strategy. In particular, the Layered Closure is used only for the first layer, and then the Complete Closure is brought into play. This choice is based on the well-known observation that pruning typically has the maximum impact for itemsets of length two - that is, the number of 2-itemsets that turn out to be large is usually a small fraction of the possible 2-itemset candidates [14]. In contrast, the impact of pruning at higher itemset lengths is comparatively small.

To put it in a nutshell, the DELTA design endeavors to achieve a reasonable compromise between the number of candidates counted and the number of database passes, since these two factors represent the primary bottle-necks in association rule generation. That our choice of compromise results in good performance is validated in the experimental study described in Section 6. 


\section{MULTI-SUPPORT INCREMENTAL MINING IN DELTA}

In the previous section, we considered incremental mining in the context, of "equi-support" environments. As mentioned in the Introduction, however, we would expect that user requirements would typically change with time, resulting in different minimum support levels across mining operations. In DELTA, we address this issue which has riot been previously considered in the literature. We expcc't that this is an important value addition given the inherent, exploratory nature of mining.

For convenience, we break up the multi-support problem into two cases: Stronger,; where the current, threshold is higher (i.e., $\sup _{\min }^{D B \cup d b}>\sup _{\min }^{D B}$ ), and Weaker, where the current, threshold is lower (i.e., $\sup _{\min }^{D B \cup d b}<s u p_{\min }^{D B}$ ). We now address each of these cases separately:

\subsection{Stronger. Support Threshold}

'The stronger support case is handled almost exactly the same way as the equi-support case, that is, as though the threshold has not chonyed. The only difference is that the following optimization is incorporated:

Initially, all itemsets which are not large w.r.t. sup min $\operatorname{midb}^{D B}$ are removed from $L^{D B}$ and the corresponding negative border is then calculated. The itemsets that are removed are not discarded completely, but, are retained separately since they may become large after counting over the incremerit $d h$. They may also be part of the computed negative border closure (lines 15-20 in Figure 1). If so, then duririg the pass over $D B$ their counts are riot measured since they arc already known. If the counts of all the itemsets in the closure are known. the pass over $D B$ becomes unnecessary

\subsection{Weaker Support Threshold}

The weaker support case is much more difficult to handle since the $L^{D B}$ set now needs to bo expanded but the identities of these additional sets cannot be deduced from the increment, $d b$. In particular, note that, Theorem 2, which DELTA relied on for pruning candidates in the equisupport case, no longer holds when the support threshold is lowered since wc cannot deduce that. a candidate is small over $D B$, just because it is not present in $L^{D B} \cup N^{D B}$.

However, it is easy to observe that the output required in the weaker threshold case is a suprset of what would be output had the support threshold not changed. This observation suggests a strategy by which the DELTA algorithm is executed as though the support, threshold had not changed, while at the same time making suitable alterations to handle the support, threshold change.

In DELTA, the above strategy is incorporated by generating extra candidates (as described below) based on the lowered support threshold. It is only for these candidates that Theorem 2 does not, hold. Hence, it is necessary to find their counts over the entire database $D B \cup d b$. This is done simultaneously while executing equi-support DELTA.

The pseudo-code for the complete algorithm is given as function DeltaLow in Figure 2, and is described in the remainder of this section. The important point to note here is that the enhanced DELTA requires only one additional pass over the entire database to produce the desired results.

\subsubsection{First Pass over the Increment}

As in the equi-support case, the counts of itemsets in $L^{D B}$ and $N^{\prime \prime \prime}$ are updated over the increment $d b$ (line 1 in Figure 2). By this, some itemsets in $N^{D B}$ may become large and some itemsets in $L^{D B}$ may become small. Let the resultant set of large itemsets (w.r.t. sup $p_{\min }^{D B}{ }^{D}$ ) be $L$. These large itemsets are extracted using the function GetLarge (line 2). Itemsets in the negativr border of $L$ with unknown counts are computed as $\operatorname{NegBorder}(L)-\left(L^{D B} \cup N^{D B}\right)$. We refer to this set, as NBetween since these itemsets are likely to have supports between $\sup _{\min }^{D B}$ and $s u p_{\min }^{D B U d b}$ (line 3). For these itemsets, Theorem 2 does not hold due to the lowered support threshold. 


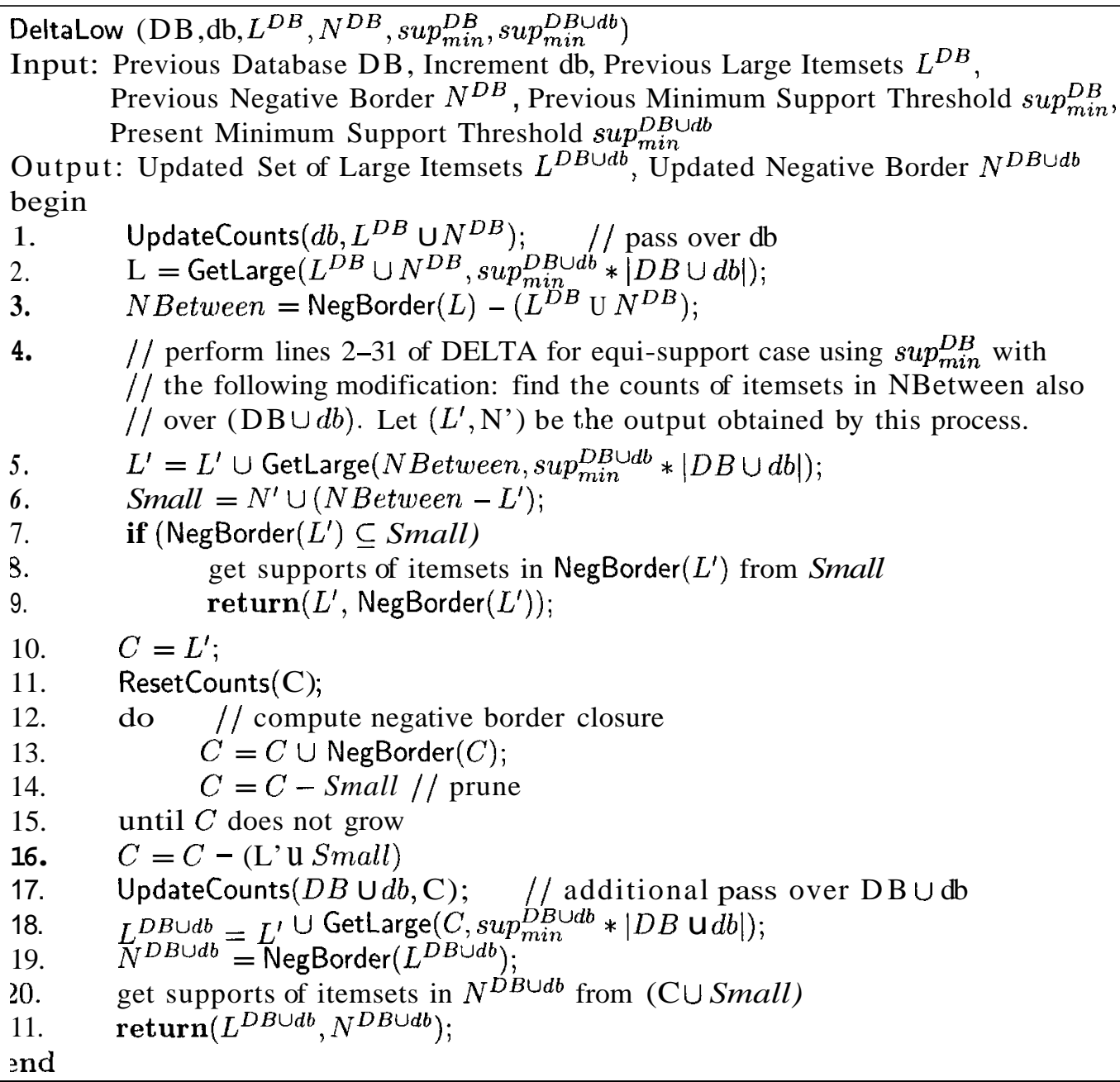

Fig. 2: DELTA for Weaker Support Threshold (DeltaLow)

\subsubsection{Remaining Passes of Equi-Support DELTA}

The remaining passes of equi-support DELTA are executed for the previous support $s u p_{\min }^{D B}$. A difference, however, is that, the counts of itemsets in NBetween over DB $\cup \mathrm{db}$ are simultaneously found (line 4).

Among the candidates generated during the remaining passes of equi-support DELTA, some may already be present in NBetween. To ensure that there is no unnecessary duplicate counting, all such common itemsets are identified and only one copy of each is retained during counting.

\subsubsection{Additional Pass over the Entire Database}

At the end of the above passes, the counts of all 1-itemsets and 2-itemsets of $L^{D B \cup d b} \cup N^{D B \cup d b}$ are available. The counts of 1-itemsets are available because $L^{D B} \cup N^{D B}$ contains all possible 1-itemsets [18], while the counts of all required 2 -itemsets are available because $L$ contains all large 1-itemsets in $\mathrm{DB} \cup d b$ and NBetween contains the immediate extensions of $\mathrm{L}$ that are not already in $\left(L^{D B} \cup N^{D B}\right)$. Therefore, it becomes possible to generate the negative border closure of all known large itemsets without encountering the "candidate explosion" problem described for the Complete Closure approach in Section 2.3.

Let $L^{\prime}$ be the set of all large itemsets whose counts are known (line 5), and let Small be the set of itemsets with known counts which are not in $L^{\prime}$ (line 6). If the counts of the negative border 
of $L^{\prime}$ are already known: then the algorithm terminates (lines 79 ). Otherwise, all the remaining extensions of $L$ ' that could become large are determined by computing the negative border closure (lines 10-16). (As in the equi-support case, we expect, that the remaining layers of the closure can be generated together since the number of 2-itemsets in $L^{\prime}$ is typically niuch smaller than the overall number of all possible 2-itemset pairs.) The itemsets of the closure are counted over the entire database (line 17), and the final set of large iternsets and its negative border are determined (lines 18-20).

\section{PREVIOUS INCREMENTAL ALGORITHMS}

In this section, we provide an overview of the algorithms that have been developed over the last two years for incremental mining of flat association rules on market basket databases.

\subsection{The FUP Algorithm}

The FUP (Fast UPdate) algorithm $[8,9,10]$ represents the first work in the area of incremental mining. It operates on an iterative basis and in each iteration makes a complete scan of the current database. In each scan, the increment is processed first and the results obtained are used to guide the mining of the original database $D B$. An important point to note about, the FUP algorithm is that it requires $k$ - passes over the entire database, where $k$ is the cardinality of the longest large itemset. Further, it does not, generate the mining results for solely the increment.

In the first pass over the increment, all the 1-itemsets are considered as candidates. At the end of this pass, the complete supports of the candidates that happen to be also large in $D B$ are known. Those which have the minimum support are retained in $L^{D B \cup d b}$. Among the other candidates. only those which were large in $d b$ can become large overall duo to Theorem 2 (Section 2). Hence they are identified and the previous database $D B$ is scanned to obtain their overall supports, thus obtaining the set of all large 1-itemsets. The candidates for the next pass are calculated using the AprioriGen function, arid the process repeats in this manner until all the large itemsets have been identified.

After FUP, algorithms that utilized the negative border information were proposed independently in [11] and [18] with the goal of achieving more efficiency in the incremental mining process. In the sequel, we will use Borders to refer to the algorithm in [11], arid TBAR. to refer to the algorithm in [18]. Since these algorithms are based on the negative border concept, they will be described in terms of the DELTA design.

\section{4..?. The Borders Algorithm}

The original Borders algorithm differs from DELTA in that it computes the entire negative border closure at one shot, that is, it uses the Complete Closure option, which could potentially result in the candidate explosion problem mentioned in Section 2.3,

A new version of the Borders algorithm was recently proposed in [5]. This version goes to the other extreme of the closure computation, adopting a Layered Closure approach. As mentioned in Section 2.3. this strategy could result, in significantly increasing the number of database passes, and may therefore be problematic for largo databases.

A variant of the new algorithm was proposed to handle multi-support mining. The applicability of this algorithm, however, is limited to the very special case of zero-size increments. that is. where the database has not changed at all between the previous arid the current mining.

Finally, like FUP, Borders also does not generate the mining results for solely the increment.

\subsection{The TBAR Algorithm}

The TBAR algorithm differs from DELTA in two major respects: First, it initially completely mines the increment $d b$, that is, $L^{d b} \cup N^{d b}$ is computed by applying the Apriori algorithm on the increment. We expect that this strategy would prove to be inefficient for large increments since the previous mining results are not used at all in this mining process. 
Second, it adopts the Complete Closure approach. The complete closure is however computed only after having mined the increment. Therefore, unlike Borders, the candidate explosion problem is unlikely to occur because more candidates can be pruned. After computing each level of the closure, itemsets in $N^{d b}$ are excluded from further candidate generation. However, even with this pruning, there are likely to be too many unnecessary candidates in TBAR, especially for skewed increments since it relies solely on the increment for its pruning.

\subsection{Other Algorithms}

Recently a new algorithm for first-time mining called CARMA was proposed in [13] where its applicability to incremental mining was also briefly mentioned. Although the algorithm is a novel and efficient approach to first-time mining, we note that it suffers from the following drawbacks when applied to incremental mining: (1) It does not maintain negative border information and hence will need to access the original database $D B$ if there are any locally large itemsets in the increment, even though these itemsets may not be globally large. (2) The shrinking support intervals which CARMA maintains for candidate itemsets are not likely to be tight for itemsets that become potentially large while processing the increment. This is because the number of occurrences of such itemsets in $D B$ will be unknown and could be as much as $\sup _{\min } *|D B|$.

An incremental mining algorithm, called MLUp, for updating "multi-level" association rules over a taxonomy hierarchy was presented in [10]. While MLUp's goal is superficially similar to the incremental hierarchical mining discussed in this paper, it has the following major differences: Firstly, a different minimum support threshold is used for each level of the hierarchy. Secondly, MLUp restricts its attention to deriving intra-level rules, that is, rules within each level. In contrast, our focus in this paper is on the formulation given in [16] where there is only one minimum support threshold and inter-level rules form part of the output.

\section{PERFORMANCE STUDY}

In the previous sections, we presented the FUP, Borders and TBAR incremental mining algorithms, apart from our new DELTA algorithm. To evaluate the relative performance of these algorithms and to confirm the claims that we have informally made about their expected behavior, we conducted a series of experiments that covered a range of database and mining workloads. The performance metric in these experiments is the total execution time taken by the mining operation. (Note that, as mentioned in Section 4, both FUP and Borders do not compute the mining results for solely the increment, and hence their execution times do not include the additional processing required to generate these results.)

\subsection{Baseline Algorithms}

We include the Apriori algorithm also in our evaluation suite to serve as a baseline indicator of the performance that would be obtained by directly using a "first-time" algorithm instead of an incremental mining algorithm. This helps to clearly identify the utility of "knowing the past".

Further, as mentioned in the Introduction, it is extremely useful to put into perspective how well the incremental algorithms make use of their "knowledge of the past", that is, to characterize the efficiency of the incremental algorithms. To achieve this objective, we also evaluate the performance achieved by the ORACLE algorithm, which "magically" knows the identities of all the large itemsets (and the associated negative border) in the current database and increment and only needs to gather their corresponding supports. Note that this idealized incremental algorithm represents the absolute minimal amount of processing that is necessary and therefore represents a lower bound ${ }^{\dagger}$ on the (execution time) performance.

The ORACLE algorithm operates as follows: For those itemsets in $L^{D B \cup d b} \cup N^{D B \cup d b}$ whose counts over $D B$ are currently unknown, the algorithm first makes a pass over $D B$ and determines these counts. It then scans $d b$ to update the counts of all itemsets in $L^{D B \cup d b} \cup N^{D B \cup d b}$. During the

\footnotetext{
tWithin the framework of the data and storage structures used in our study.
} 


\begin{tabular}{|c|l|l|}
\hline Parameter & Meaning & Values \\
\hline$N$ & Number of items & 1000 \\
$T$ & Mean transaction length & 10 \\
$L$ & Number of potentially large itemsets & 2000 \\
$I$ & Mean length of potentially large itemsets & $\mathbf{3}$ \\
$D$ & Nuniber of transactions in database $D B$ & $4 \mathrm{M}(200 \mathrm{MB}$ disk occupancy) \\
$d$ & Number of transactions in increment $d b$ & $1 \%, 10 \%, 50 \%, 100 \%$ of $D$ \\
$S$ & Skew of increment $d b$ (w.r.t. $D B$ ) & Identical, Skewed \\
$P i s$ & Prob. of changing large itemset identity & 0.33 (for Skewed) \\
$p_{i t}$ & Prob. of changing item identity & 0.50 (for Skewed) \\
\hline
\end{tabular}

Table 2: Parameter Table

\begin{tabular}{|c|l|l|}
\hline Parameters & Meaning & Values \\
\hline$R$ & Number of roots & 250 \\
$L$ & Number of levels & 4 \\
$F$ & Fanout & 5 \\
$D$ & Depth-ratio & 1 \\
\hline
\end{tabular}

Table '3: Taxonomy Parameter Table

pass over $d h$, it also determines the counts within $d b$ of itemsets in $L^{d b} \cup N^{\text {“. }}$. Duplicate candidates are avoided by retaining only one copy of each of them. So, in the worst case, it rieeds to make one pass over the previous database and one pass over the increment.

For evaluating the performance of DELTA on hierarchical databases, we compared it with Cumulate and ORACLE as no previous incremental algorithms are available for comparison. We chose Cumulate among the algorithms proposed in [16] since it performed the best on most of our workloads. The hierarchical databases were generated using the same technique as in [16].

\subsection{Database Generation}

The databases used in our experiments were synthetically generated using the technique described in [3] and attempt to mimic the customer purchase behavior seen in retailing environments. The parameters used in the synthetic generator are described in Table 2. These are similar to those used in [3] except that the size and skew of the increment are two additional parameters. Since the generator of [3] does not include the concept of an increment, we have taken the following approach, similar to [8]: The increment is produced by first generating the entire $D B \cup d b$ and then dividing it into $D B$ and $d b$.

Additional parameters required for the taxonomy in our experiments on hierarchical databases are shown in Table 3. The values of these parameters are identical to those used in [16].

\subsubsection{Data Skew Generation}

The above method will produce data that is identically distributed in both $D B$ and $d b$. However, as mentioned earlier, databases often exhibit temporal trends resulting in the increment perhaps having a different distribution than the previous database. That is, there may be significant changes in both the number and the identities of the large itemsets between $D B$ and $d b$. To model this "skew" effect, we modified the generator in the following manner: After $D$ transactions are produced by the generator, a certain percentage of the potentially large itemsets are changed. A potentially large itemset is changed as follows: First, with a probability determined by the parameter $p_{i s}$ it is decided whether the itemset has to be changed or not. If change is decided, each item in the itemset is changed with a probability determined by the parameter $p_{i t}$. 
The item that is used to replace the existing item is chosen uniformly from the set of those items that are not already in the itemset. After the large itemsets are changed in this manner, $d$ number of transactions are produced with the new modified set of potentially large itemsets.

\subsection{Itemset Data Structures}

In our implementation of the algorithms, we generally use the hashtree data-structure [3] as a container for itemsets. However, as suggested in [2], the 2-itemsets are not stored in hashtrees but instead in a 2-dimensional triangular array which is indexed by the large 1-itemsets. It has been reported (and also confirmed in our study) that adding this optimization results in a considerable improvement in performance. All the algorithms in our study are implemented with this optimization.

\subsection{Overview of Experiments}

We conducted a variety of experiments to evaluate the relative performance of DELTA and the other mining algorithms. Due to space limitations, we report only on a representative set here. In particular, the results are presented for the workload parameter settings shown in Table 2 for our experiments on non-hierarchical (flat) databases.

The parameters settings used in our experiments on hierarchical databases are identical except for the number of items $(N)$ and the number of potentially large itemsets $(L)$ which were both set to 10000 . The specific values of additional parameters required for the taxonomy are shown in Table 3.

The experiments were conducted on an UltraSparc 170E workstation running Solaris 2.6 with $128 \mathrm{MB}$ main memory and a $2 \mathrm{~GB}$ local SCSI disk. A range of rule support threshold values between $\mathbf{0 . 3 3 \%}$ and $2 \%$ were considered in our equi-support experiments.

The previous database size was always kept fixed at 4 million transactions. Along with varying the support thresholds, we also varied the size of the increment $d b$ from 40,000 transactions to 4 million transactions, representing an increment-to-previous database ratio that ranges from $1 \%$ to $100 \%$. For our experiments on hierarchical databases, the performance was measured only for supports between $0.75 \%$ and $2 \%$ since for lower supports, the running time of all the algorithms was in the range of several hours.

Two types of increment distributions are considered: Identical where both $D B$ and $d b$ have the same itemset distribution, and Skewed where the distributions are noticeably different. For the Skewed distribution for which results are reported in this paper, the $p_{i s}$ and $p_{i t}$ parameters were set to $\mathbf{0 . 3 3}$ and 0.5 as mentioned in Table 2 . With these settings, at the 0.5 percent support threshold and a 10\% increment, for example, there are over 700 large itemsets in $d b$ which are not large in $D B$, and close to 500 large itemsets in $D B$ that are not large in $d b$.

We also conducted experiments wherein the new minimum support threshold is different from that used in the previous mining. The previous threshold was set to $0.5 \%$ and the new threshold was varied from $0.2 \%$ to $1.5 \%$. Therefore, both the Stronger Threshold and Weaker Threshold cases outlined in Section 2 are considered in these experiments.

\section{EXPERIMENTAL RESULTS}

In this section, we report on the results of our experiments comparing the performance of the various incremental mining algorithms for the dynamic basket database model described in the previous section.

\subsection{Experiment 1: Flat / Equi-Support / Identical Distribution}

Our first experiment considers the equi-support situation with identical distribution between $D B$ and $d b$ on flat databases. For this environment, the execution time performance of all the mining algorithms is shown in Figures 3a-d for increment sizes ranging from $1 \%$ to $100 \%$. 


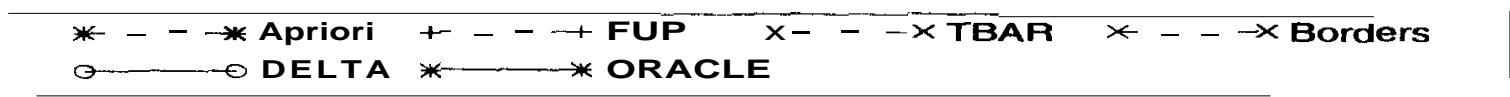

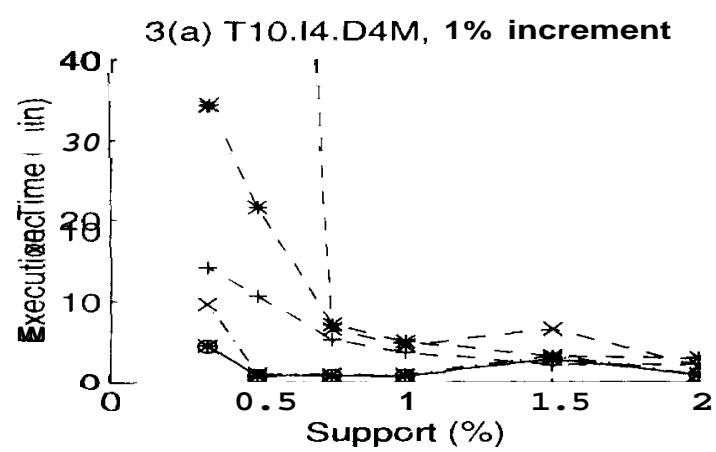

3(c) T10.14.D4M, 50\% increment

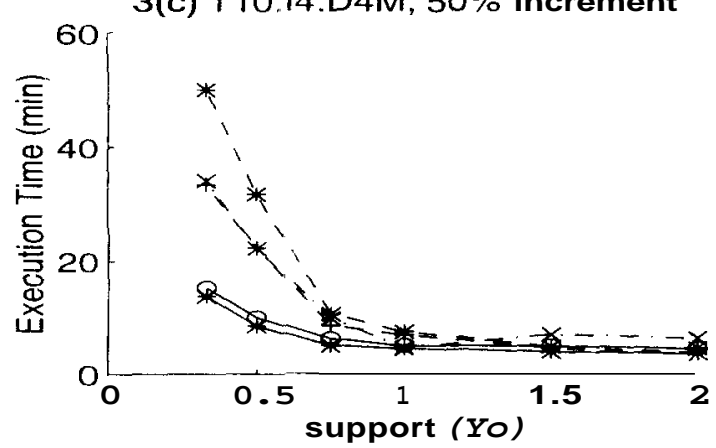

3(b) TIO.14.D4M, 10\% increment
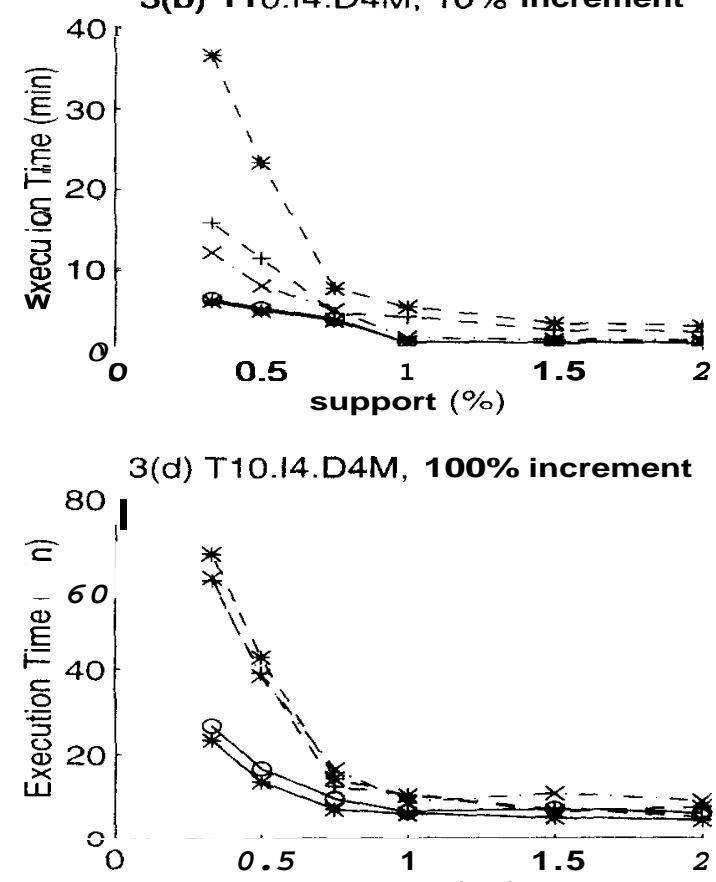

3(d) T10.14.D4M, 100\% increment

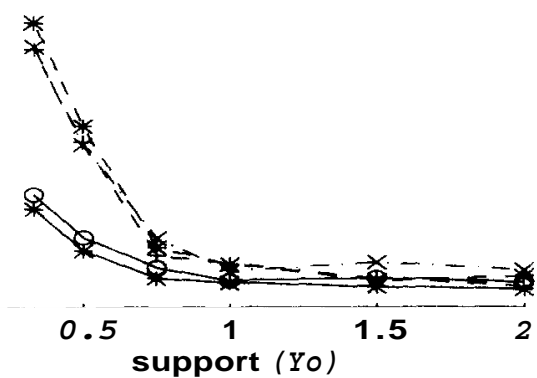

Fig. 3: Flat / Equi-Support / Identical Distribution

Focusing first on FUP, we see in Figure 3 that for all the increment sizes and for all the support factors, FUP performs better than or almost the same as Apriori. Moving on to TBAR, we observe that it outperforms both Apriori and FUP at small increment sizes and low supports. At high supports, however. it is slightly worse than Apriori due to the overhead of maintaining the negative border information. As the increment size increases, TBAR's performance becomes progressively degraded. This is explained as follows: Firstly, TBAR updates the counts of itemsets in $L^{D B} \cup N^{D B}$ over $d b$ these itemsets are precisely the same as the set, of all candidates generated in running Apriori over $D B$. Secondly, it, performs a coniplete Apriori-based mining over $d b$. When $|d b|=$ $|D B|$, the total cost of these two factors is the same as the total cost incurred by the Apriori algorithm. However. TBAR finally loses out because it needs to make a further pass over $D B$.

Turning our attention to Borders, we find in Figure 3a, which corresponds to the 1 percent increment, that while for much of the support range its performance is similar to that of FUP and TBAR, there is a sharp degradation in performance at a support of 0.75 percent. The reason for this is the "candidate explosion" problem described earlier in Section 4. This was confirmed by Ineasuring the number of candidates for supports of 1 percent and 0.75 percent - in the former case, it was a little over 1000 whereas in the latter, it had jumped to over 30000 !

The above candidate explosion problem is further intensified when the increment size is increased, to the extent that, its performance is an order of magnitude worse than the other algorithms therefore we have not shown Borders performance in Figures $3 \mathrm{~b}-\mathrm{d}$.

Finally, considering DELTA, we find that it, significantly outperforms all the other algorithms at lower support, thresholds for all the increment sizes. In fact,, in this region, the performance of DELTA almost coincides with that of ORACLE. The reason for the especially good performance here is the following - low support values result in tighter values of $k$, the maximal large itemset size, leading to correspondingly more iterations for FUP over the previous database $D B$, and for TBAR over the increment, $d b$. In contrast, DELTA requires only three passes over the increment 


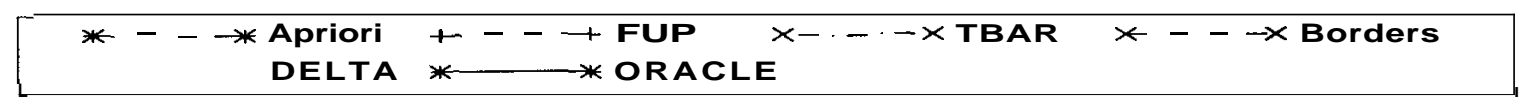

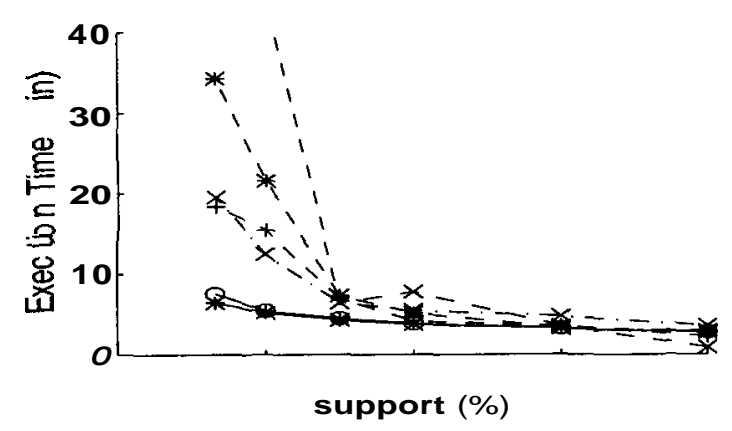

4(c) T10.14.D4M, 50\% increment

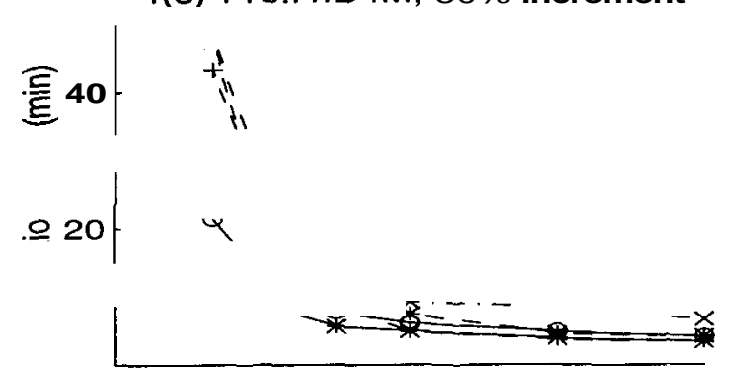

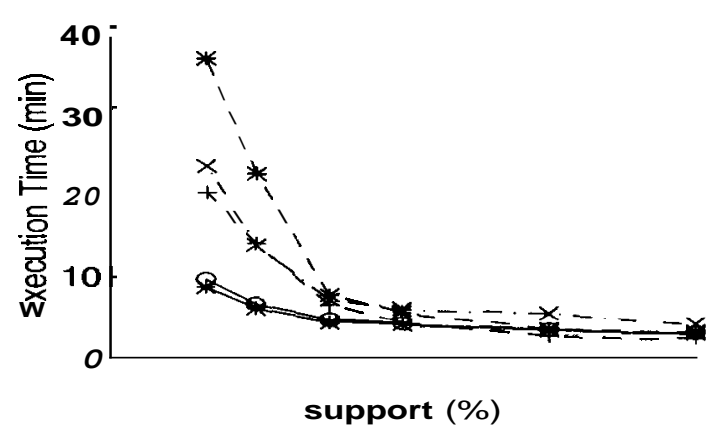

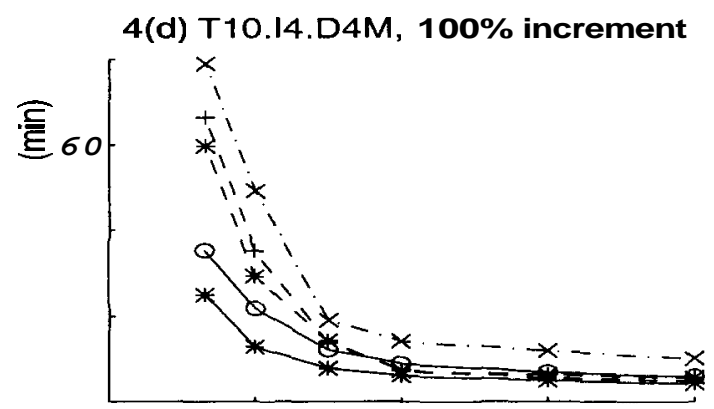

Fig. 4: Flat / Equi-Support / Skewed Distribution

and one pass over the previous database. Further, because of its pruning optimizations, the number of candidates to be counted over the previous database $D B$ is significantly less as compared to TBAR - for example, for a support threshold of 0.5 percent and a $50 \%$ increment (Figure 3c), it is smaller by a factor of two.

We note that the marginal non-monotonic behavior in the curves of TBAR, Borders, DELTA and ORACLE at low increment sizes is due to the fact that only sometimes do they need to access the original database $D B$ and this is not a function of the minimum support threshold.

\subsection{Experiment 2: Flat / Equi-Support / Skewed Distribution}

Our next, experiment considers the Skewed workload environment, all other parameters being the same as that of the previous experiment. The execution time performance of the various algorithms for this case is shown in Figures $4 a-d$. We see here that the effect of the skew is pronounced in the case of both TBAR and Borders, whereas the other algorithms (including DELTA) are relatively unaffected.

The effect of skew is noticeable in the case of TBAR since it relies solely on the increment to prune candidates from its computation of the closure and therefore many unnecessary candidates are generated which later prove to be small over the entire database. Borders, on the other hand, is affected because the number of 1-itemsets that are in the promoted border tends to increase when there is skew. For instance, for a minimum support of $0.33 \%$ and an increment of $10 \%$, there were nine 1 -itemsets among the promoted borders and the number of large itemsets was 4481, resulting in over 2 million candidates.

In contrast to the above, Apriori and FUP are not, affected by skew since the candidates that they generate in each pass are determined only by the overall large itemsets, and not by the large itemsets of the increment. 


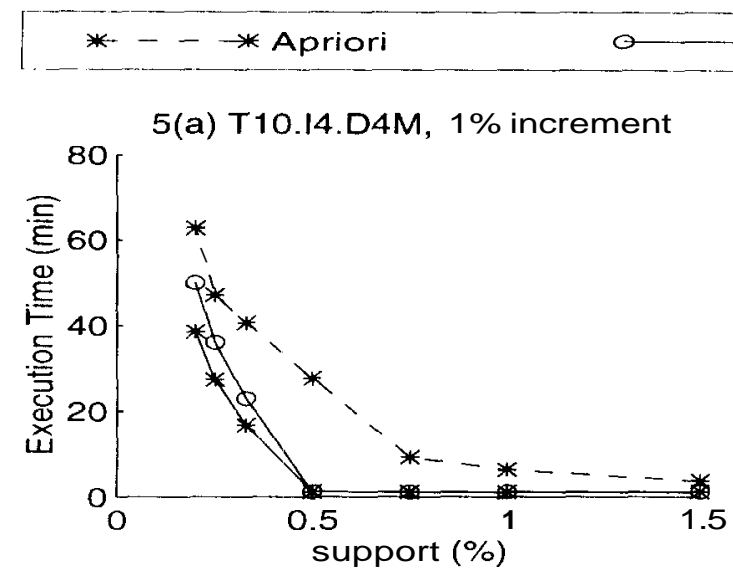

DELTA

* - * ORACLE
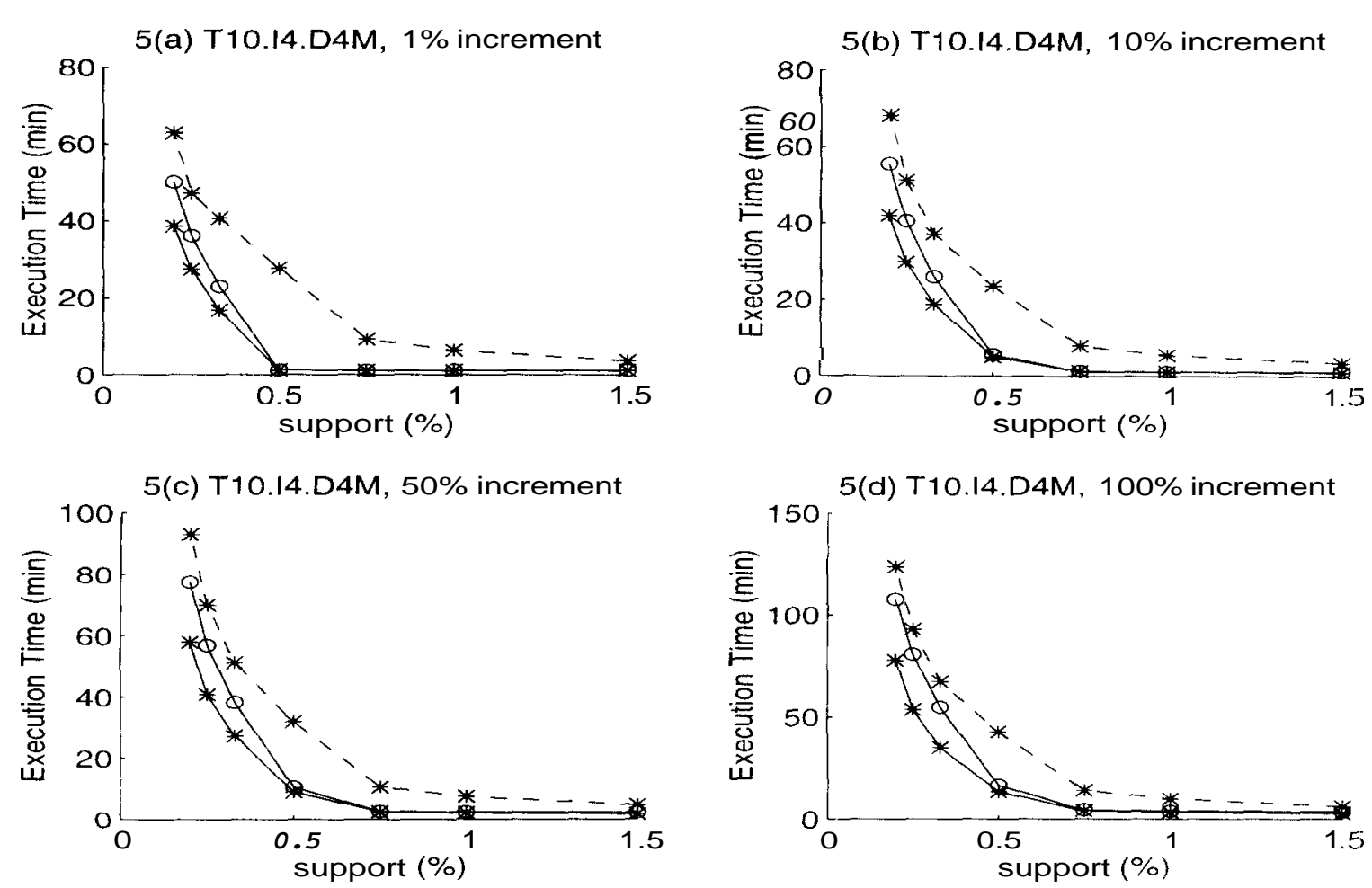

Fig. 5: Flat / Multi-Support / Identical Distribution [Previous Support, $=0.5 \%$

DELTA is not as affected by skew as TBAR since it utilizes the complete negative border information to prune away candidates. That is, all itemsets which are known to be small either over $D B \cup d h$ or over $d b$ are pruned away during closure generation, and not merely those candidates which are small over $d b$. Hence, DELTA is relatively stable with respect to data skew. As in the Identical distribution case, it can be seen in Figures $4 a-b$ that for small increment sizes. its performance almost, coincides with that of ORACLE. It however degrades to some extent, for large skewed increments because of two reasons: (1)the number of itemsets in $L^{D B}-L^{D B \cup} \cup l$ increases, resulting in more unnecessary candidates being updated over $d b$, and (2) the number of itemsets in $L^{D B \cup d b}-L^{D B}$ increases, resulting in more promoted borders followed by more candidates over $D B$. Even in these latter cases it is seen to perform considerably better than other algorithms. For example, for a minimum support, of $0.33 \%$ and an increment of $100 \%$. its performance is more than twice as good as that of TBAR.

\subsection{Experiment 3: Flat / Multi-Support / Identical Distribution}

The previous experiments modeled equi-support environments. We now move on to considering multi-support environments. In these experiments, we compare the performance of DELTA with that of Apriori and ORACLE only since, as mentioned earlier, FUP, TBAR. and Borders do not handle the multi-support case.

In this experiment, we fixed the initial support to be $0.5 \%$ and the new support was varied between $0.2 \%$ and $1.5 \%$, thereby covering both the Weaker Threshold arid Stronger Threshold possibilities. For this environment, Figures 5a-d show the performance of DELTA relative to that of Apriori for the databases where the distribution of the increments is Identical to that, of the previous database.

We note here that at either end of the support spectrum, DELTA performs very similarly to Apriori whereas in the "middle band" it does noticeably better, especially for moderate increment 


* - * * Apriori $\quad$ o DELTA $\cdots$ ORACLE
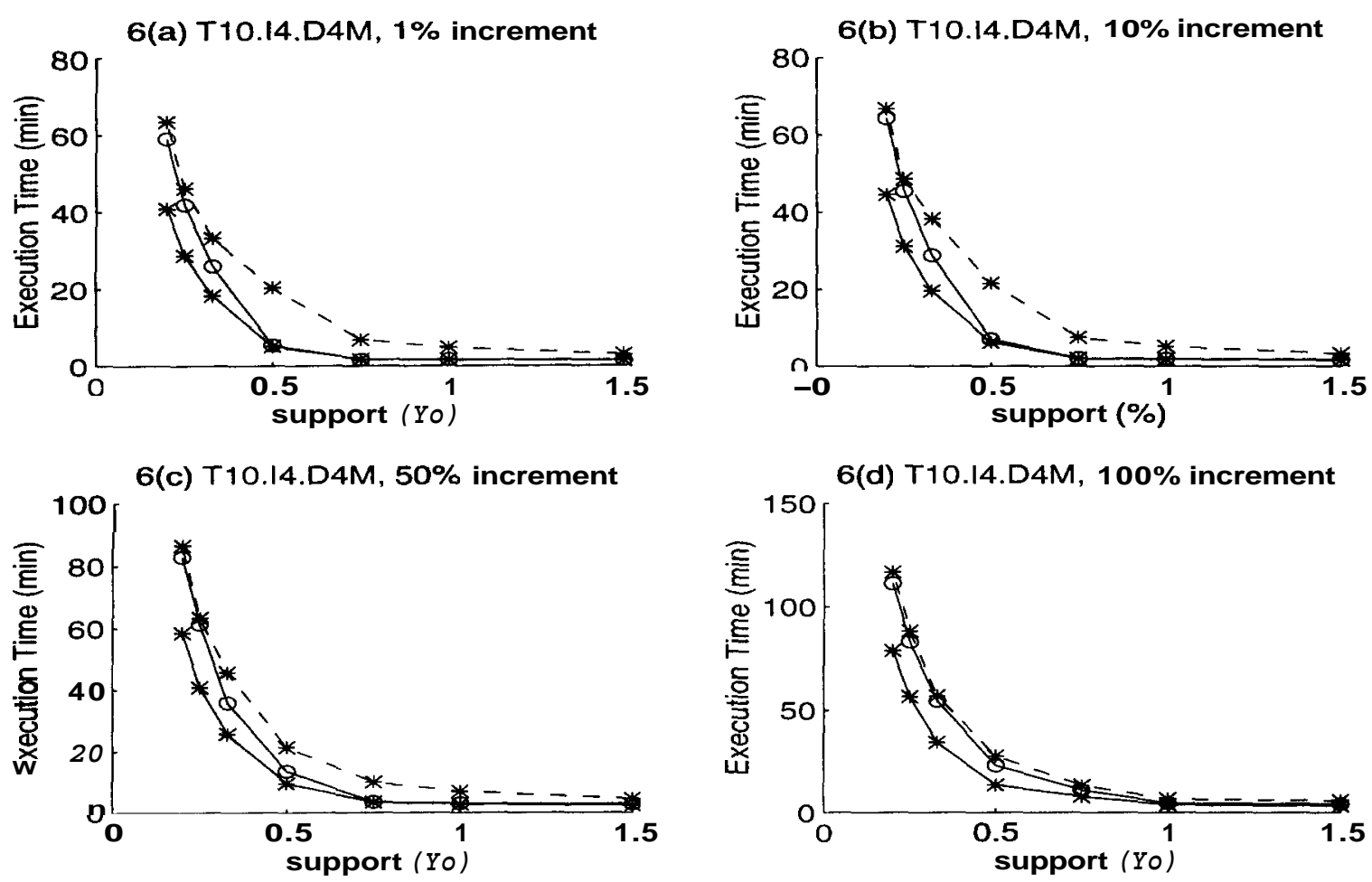

Fig. 6: Flat / Multi-Support / Skewed Distribution [Previous Support $=0.5 \%$ ]

sizes (Figures 5a-b). In fact, the performance gain of DELTA is maximum when the new minimum support threshold is the same as the previous threshold and tapers off when the support is changed in either direction. At very low support thresholds, the number of large itemsets increases exponentially, and therefore the number of candidates generated in the negative border closure in DELTA will be a few more than the number of candidates generated in Apriori. Most of the candidates will have support less than the previous minimum threshold, and hence all of them have to be counted over the previous database. Therefore, the performance of DELTA approaches that of Apriori in the low support region. In the high support region, on the other hand, most of the candidates do not turn out to be large and hence both algorithms perform almost the same amount of processing.

\subsection{Experiment 4: Flat / Multi-Support / Skewed Distribution}

Our next experiment evaluates the same environment as that of the previous experiment, except that the distribution of the increments is Skewed with respect to the original database. The execution time performance for this case is shown in Figures 6a-d. We see here that the relative performance of the algorithms is very similar to that seen for the Identical workload environment. Further, as in the equi-support skewed case (Experiment 2), DELTA is stable with respect to skew since it uses information from both $D B$ and $d b$ to prune away candidates. Only when the increment size is $100 \%$ do we notice some degradation in the performance of DELTA. However, it performs slightly better than Apriori even for this large increment.

\subsection{Experiment 5: Hierarchical / Equi-Support / Identical Distribution}

The previous experiments were conducted on flat databases. We now move on to experiments conducted on hierarchical databases. In these experiments, we compare the performance of DELTA 

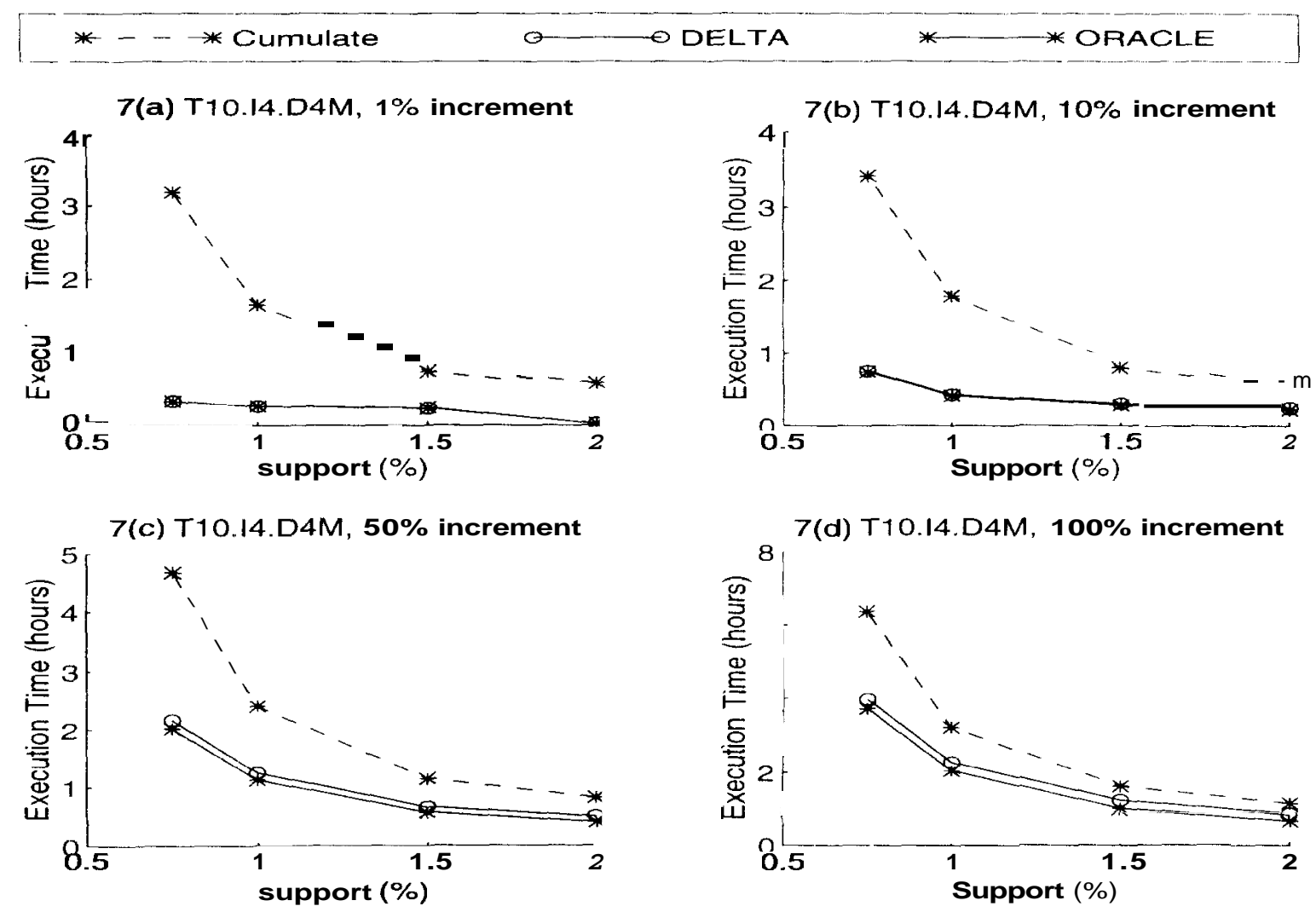

Fig. 7: Hierarchical / Equi-Support / Identical Distribution

with that of Cumulate and ORACLE only since, as mentioned earlier, no incremental algorithms are available for comparison. The execution time performance of the various algorithms for this case is shown in Figures $7 \mathrm{a}-\mathrm{d}$. Note that the time taken to complete mining is measured in hours here as cornpared to the minutes taken in the previous experiments. The reason for this large increase is that the number of large itemsets is much more (about, 10--15 times) - this is because itemsets can be formed both within and across levels of the item taxonomy graph.

For all support, thresholds and database sizes, we find that DELTA significantly outperforms Curnulate, and is in fact very close to ORACLE. We see that DELTA exhibits a huge performaricc gain over Cumulate, upto as much us 9 times at the $1 \%$ increment and $0.75 \%$ support, threshold, and as much as 3 times on average. In fact, the performance of DELTA is seen to overlap with that of ORACLE for small increments (Figures $7 a-b$ ). The reason for this is the number of candidates in DELTA over both $d b$ and $D B$ were only marginally more than that in ORACLE. This is again because the set, of large itemsets with its negative border is relatively stable, arid DELTA prunes away most of the unnecessary candidates in its second pass over the increment.

Due to space constraints, the experimental results for hierarchical databases where the increment's distribution is Skewed, as also the niulti-support environments, are not presented herr. Thev are available in [15] and are similar in nature to those presented earlier in this paper for flat. databases.

\section{CONCLUSIONS}

We considered the problem of incrementally mining association rules on market basket databases that, have been subjected to a significant number of updates since their previous mining exercise. Instead of mining the whole database again from scratch, we attempt to use the previous mining results, that is, knowledge of the itemsets which are large in the previous database, their negative 
border, and their associated supports, to efficiently identify the same information for the updated database.

We proposed a new algorithm called DELTA which is the result of a synthesis of existing algorithms, designed to address each of their specific limitations. It guarantees completion of mining in three passes over the increment and one pass over the previous database. This compares favorably with previously proposed incremental algorithms like FUP and TBAR wherein the number of passes is a function of the length of the longest large itemset. Also, DELTA does not suffer from the candidate explosion problem associated with the Borders algorithm owing to its better pruning strategy.

DELTA's design was extended to handle multi-support environments, an important issue not previously addressed in the literature, at a cost of only one additional pass over the current database.

Using a synthetic database generator, the performance of DELTA was compared against that of FUP, TBAR and Borders, and also the two baseline algorithms, Apriori and ORACLE. Our experiments showed that for a variety of increment sizes, increment distributions and support thresholds, DELTA performs significantly better than the previously proposed incremental algorithms. Infact, for many workloads its performance approached that of ORACLE, which represents a lower bound on achievable performance, indicating that DELTA is quite efficient in its candidate pruning process. Also, while the TBAR and Borders algorithms were sensitive to skew in the data distribution, DELTA was comparatively robust.

In the special scenario where no pass over the previous database is required since the new results are a subset of the previous results, DELTA's performance is optimal in that it requires only one pass over the increment whereas all the other algorithms either are unable to recognize the situation or require multiple passes over the increment.

Finally, DELTA was shown to be easily extendible to hierarchical association rules, while maintaining its performance close to ORACLE. No prior work exists on extending incremental mining algorithms to handle hierarchical rules.

In summary, DELTA is a practical, robust and efficient incremental mining algorithm. In our future work, we plan to extend the DELTA algorithm to handle quantitative rules [17] and also to develop incremental algorithms for sequence [4] and classification rules [6].

Acknowledgements - This work was supported in part by research grants from the Department of Science and Technology and the Department of Biotechnology, Government of India. We thank Shiby Thomas and Rajeev Rastogi for their expert feedback and suggestions on the material presented here.

\section{REFERENCES}

[1] R. Agrawal, T. Imielinski, and A. Swami. Mining association rules between sets of items in large databases. In Proceedings of the 22nd A CM SIGMOD International Conference on Management of Data, Washingtion, D.C, pp. 207-216, ACM Press (1993).

[2] R. Agrawal and J. Shafer. Parallel mining of association rules: design, implementation and experience. Technical Report RJ10004, IBM Almaden Research Center, San Jose, CA 95120 (1996).

[3] R. Agrawal and R. Srikant. Fast algorithms for mining association rules. In Proceedings of the 20th International Conference on Very Large Databases (VLDB), Santiago de Chile, Chile, pp. 487-499, Morgan Kaufmann (1994).

[4] R. Agrawal and R. Srikant. Mining sequential patterns. In Proceedings of the 11th International Conference on Data Engineering (ICDE), Taipei, Taiwan, pp. 3-14. IEEE Computer Society (1995).

[5] $\boldsymbol{Y}$.Aumann, R. Feldman, O.Lipsttat, and H. Manilla. Borders: an efficient algorithm for association generation in dynamic databases. In Journal of Intelligent Information Systems, pp. 61-73, Kluwer Academic Publishers, http://www.isse.gmu .edu/JIIS/1999).

[6] L. Breiman, J. Friedman, R. Olshen, and C. Stone. Classification and Regression Trees. Belmont: Wadsworth (1984).

[7] S. Brossette, A. Sprague, J. Hardin, K. Waites, W. Jones, and S. Moser. Association rules and data mining in hospital infection control and public health surveillance. In Journal of the American Medical Informatics Association, pp. 373-381, American Medical Informatics Association, http://www.jamia.org/ (1998).

[8] D. Cheung, J. Han, V. Ng, and C. Wong. Maintenance of discovered association rules in large databases: an incremental updating technique. In Proceedings of the 12th International Conference on Data Engineering $(I C D E)$, New Orleans, Louisiana, pp. 106-114, IEEE Computer Society (1996). 
[9] D. Cheung, S. Lee, arid B. Kao. A general incremental technique for maintaining discovered association rules. In Proceedings of the 5th International Conference on Database Systems for Advanced Applications (DASFAA), Melbourne, Australia, pp. 185-194, World Scientific (1997).

[10] D. Cheung, 'T. Vincent, and W. Benjamin. Maintenance of discovered knowledge: a case in multi-level association rules. In Proceedings of the 2nd International Conference on Knowledge Discovery and Data Minina (KDD), Portland, Oregon, pp. 307-310, AAAI Press (1996).

[11] R. Feldman, Y. Aumann, A. Amir. and H. Mannila. Efficient algorithms for discovering frequent, sets in incremental databases. In Proceedings of SIGMOD Workshop on Research Issues in Data Mining and Knowledge Discovery, Tucson. Arizona (1997).

[12] M. Garofalakis. S. Ramaswamy, R. Rastogi, and K. Shim. Of crawlers, portals, mice, and men: is there more to mining the wob? In Proceedings of the 28th ACM SIGMOD International Conference on Management of Data, Philadephia, Pennsylvania, p. 504, ACM Press (1999).

[13] (. Hidber. Online association rule mining. In Proceedings of the 28th A CM SIGMOD international Conference on Management of Data, Philadephia, Pennsylvania, pp. I45-156, ACM Press (1999).

[Id] J. Park, M. Chen, and P. Yu. An effective hash-based algorithm for mining association rules. In Proceedings of /he 24th A CM SIGMOD International Conference on Management of Data, Sari .Jose, California, pp. 175186, ACM Press (1995).

[15] V. Pudi arid J.R. Itaritsa. Quantifying the Utility of the Past 271 Mining Large Databases. Technical Report. TR-2000-01, DSL, Indian Institute of Science, http://dsl.serc.iisc.ernet.in/pub/TR/TR-2000-01.ps (2000).

[16] R. Srikant and R. Agrawal. Mining generalized association rules. In Proceedings of the 21st International Conference on Very Large Databases (VLDB), Zürich, Switzerland, pp. 407-419, Morgan Kaufmann (1995).

[17] R. Srikant anti 11. Agrawal. Mining quantitative association rules in large relational tables. In Proceedings of the 25th ACM SIGMOD International Conference on Management of Data, Montreal, Canada, pp. 1-12, ACM Press (1996)

[18] S. Thomas, S. Bodagala, K. Alsabti, arid S. Rnnka. An efficient algorithm for the incremental updation of association rules in large databases. In Proceedings of the isd International Conference on Knowledge Iiscovery and Data Mining (KDD), Newport Beach, California, pp. 263 -266, A A AI Press (1997).

[19] 11. Toivonen. Sampling large databases for association rules. In Proceedings of the 22nd Internatzonal Conference on Very Large Databases (VLRB), Bombay, India, pp. 134-145. Morgan Kaufmann (1996). 